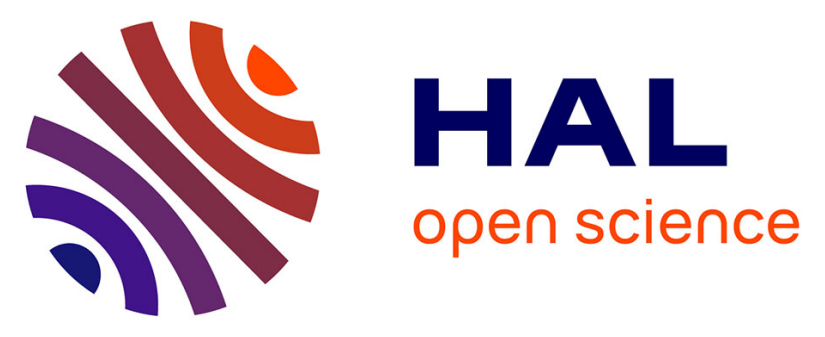

\title{
Profiler for Smartphone Users Interests Using Modified Hierarchical Agglomerative Clustering Algorithm Based on Browsing History
}

\author{
Priagung Khusumanegara, Rischan Mafrur, Deokjai Choi
}

\section{To cite this version:}

Priagung Khusumanegara, Rischan Mafrur, Deokjai Choi. Profiler for Smartphone Users Interests Using Modified Hierarchical Agglomerative Clustering Algorithm Based on Browsing History. 3rd International Conference on Information and Communication Technology-EurAsia (ICT-EURASIA) and 9th International Conference on Research and Practical Issues of Enterprise Information Systems (CONFENIS), Oct 2015, Daejon, South Korea. pp.89-96, 10.1007/978-3-319-24315-3_9 . hal01466240

\author{
HAL Id: hal-01466240 \\ https://hal.inria.fr/hal-01466240
}

Submitted on 13 Feb 2017

HAL is a multi-disciplinary open access archive for the deposit and dissemination of scientific research documents, whether they are published or not. The documents may come from teaching and research institutions in France or abroad, or from public or private research centers.
L'archive ouverte pluridisciplinaire HAL, est destinée au dépôt et à la diffusion de documents scientifiques de niveau recherche, publiés ou non, émanant des établissements d'enseignement et de recherche français ou étrangers, des laboratoires publics ou privés.

\section{(c)(1)}

Distributed under a Creative Commons Attribution| 4.0 International License 


\title{
Profiler for Smartphone Users Interests Using Modified Hierarchical Agglomerative Clustering Algorithm Based on Browsing History
}

\author{
Priagung Khusumanegara, Rischan Mafrur, and Deokjai Choi \\ Department of Electronics \& Computer Engineering \\ Chonnam National University \\ 77 Yongbong-ro, Buk-guGwangju, South Korea \\ \{priagung.123, rischanlab\}@gmail.com, dchoi@cnu.ac.kr
}

\begin{abstract}
Nowadays, smartphone has been a life style for many people in the world and it has become an indispensable part of their live. Smartphone provides many applications to support human activity which one of the applications is web browser applications. People spend much time on browsing activity for finding useful information that they are interested on it. It is not easy to find the particular pieces of information that they interested on it. In this paper, userprofiler is presented as way of providing smartphone users with their interest based on their browsing history. In this study, we propose a Modified Hierarchical Agglomerative Clustering algorithm that uses filtering category groups on a server-based application to aid provides smartphone user profile for interests-focused based on browsing history automatically. Based on experimental results, the proposed algorithm can measure degree of smartphone user interest based on browsing history of web browser applications, provides smartphone users interests profile and also outperforms the C4.5 algorithm in execution time on all memory utilization.
\end{abstract}

Key words: Smartphone, user interests, Modified Hierarchical Agglomerative Clustering

\section{Introduction}

Today, many vendors such as Google and Yahoo store historical data in a users' browser to understand the type page that user is visiting. This information is used to show ads that might appeal to users based on their inferred interest categories. For example, if a user browses many sport-related websites displaying AdSense ads or watch sport-related videos on YouTube, it means Google and Yahoo may associate a sport interest category with their history and show the user sport-related ads. Information about user interests is useful both for users and service providers, user can easily find the information that they needed so they do not spend time to find it and in point of view service providers, they can also easily to provide advertisement and recommendation to the users who use their service. It is not easy to find the particular pieces of information that users interested on it. In this paper, we implement a serverbased application to provide smartphone user profile based on browsing history of 
web browser application. We propose a Modified Hierarchical Agglomerative Clustering algorithm that is inspired by Hierarchical Agglomerative Clustering algorithm. Our method can automatically provide smartphone user profile for interests-focused based on browsing history of web browser applications. First, we extract the useful information of historical web browser applications from smartphone users. Second, we use a distance function to calculate similarity distances between the extracted data. Third, we use Modified Hierarchical Agglomerative Clustering algorithm that use filtering category groups to provide smartphone user profile for interests-focused. The reminder of this paper is structured follows. The section 2 describes the previous studies. The data extraction and user profiling algorithm is presented in section 3 . We then show the experimental results and evaluations of our work in Section 4. Finally, we conclude our findings and suggestions for future research in section 5 .

\section{Related Work}

In this section, we will review some existing works on web log data mining. Previous researchers have investigated how to generate user profile based on web server data logs using various data mining technique. Most of the approaches concerned on user classification (supervised) method and clustering (unsupervised) method based on useful information from web server data logs. In Jian's et al. work [1], classification (supervised) method is used to predict users' gender and age from web browsing behavior. Santra et al. [2] research about identification interested users using naïve Bayesian classification based on web log data and also comparison between decision tree algorithm C4.5 and Naïve Bayesian Classification algorithm for identifying interested user. JinHuaXu et al. [3] used KMeans algorithm for clustering web user based on web data logs. Xia Min-jie et al. [4] research using clustering technique based on web logs and users' browsing behavior to implement an ecommerce recommendation system. Neetu et al. [5] used classification technique to predict kid's behavior based on collected internet logs. Li et al. [6] focused on web log data processing to analyze and research the user's behavior. Shuqing et al. [7] provided novel algorithm to extract user's interest based on web log data and describes including long term interest and short term interest. Tsuyoshi et al. [8] described in his paper a method for clarifying user's interests based on an analysis of the site keyword graph. In this paper, we concern on how to provide smartphone user profile automatically using Modified Hierarchical Agglomerative Clustering Algorithm based on their historical logs of web browser applications in smartphone.

\section{Data Extraction and User Profiling}

In this section, we will describe about data extraction and each process to provide smartphone user profile for interest-focused based on browsing history of web browser applications. 


\subsection{Data Extraction}

In this work, we use browsing history data of 30 smartphone users that is collected during one month. In this study, we develop an android application that can be used to collect browsing history from all browser applications and then we install that application on each user's smartphone. The structure of collected data from user's smartphone is shown in Table 1.

Table 1. The Example of Collected Data

\begin{tabular}{lll}
\hline User ID & Visit Time & URL \\
\hline 10 & 1399652396.55 & http://www.kakao.com/fightingkorea \\
7 & 1399809440.79 & http://cyber.kepco.co.kr/ckepco/ \\
1 & 1400251354.06 & http://asked.kr/ask.php?id=1927949 \\
5 & 1399553574.34 & http://m.winixcorp.com/ \\
5 & 1399637818.62 & http://www.dalkomm.com/ \\
\hline
\end{tabular}

Based on the Table 1, each row of collected data represents the URLs that the user visits. Attributes of the data include user ID, visit time, and URL data. A URL (Uniform Resource Locator) is the unique address of documents and other resources on the World Wide Web. The first part of URL structure is called a protocol identifier which indicates what protocol that is used, and the second part is called a resource name which specifies the IP address or domain name where the resource is located. In our work, we extract collected data to derive a resource name part of URL structure which is useful information to analyze user interests and after that the Modified Hierarchical Agglomerative Clustering algorithm is assigned to provide smartphone user profile for interest-focused.

\subsection{Modified Hierarchical Agglomerative Clustering Algorithm}

In this study, we have Modified Hierarchical Agglomerative Clustering to aid in providing smartphone user profile for interest-focused. We use Levenshtein distance function to measure minimum distance between two extracted URL data. We use Levenshtein distance to measure minimum distance between two extracted URL data. Levenshtein distance between two extracted URL data $\operatorname{url}_{1}, u r l_{2}$ is given by $\operatorname{dist}_{u r l_{1}, \operatorname{url}_{2}}\left(\left|\operatorname{url}_{1}\right|,\left|u r l_{2}\right|\right)$, where,

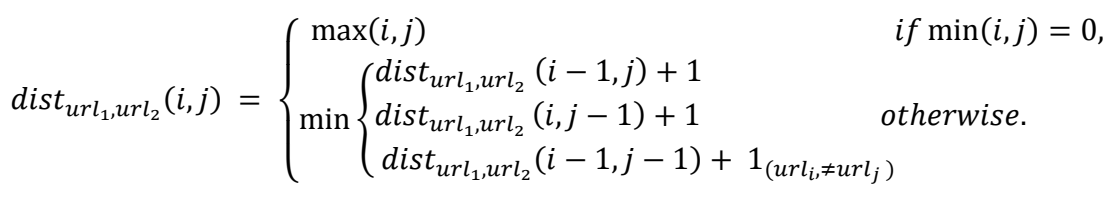

Where, $1_{\left(u r l_{i}, \neq u r l_{j}\right)}$ is the indicator function equal to 0 when $u r l_{i}=u r l_{j}$ and equal to 1 otherwise. 
The Modified Hierarchical Agglomerative Clustering algorithm that is implemented in our work is following below.

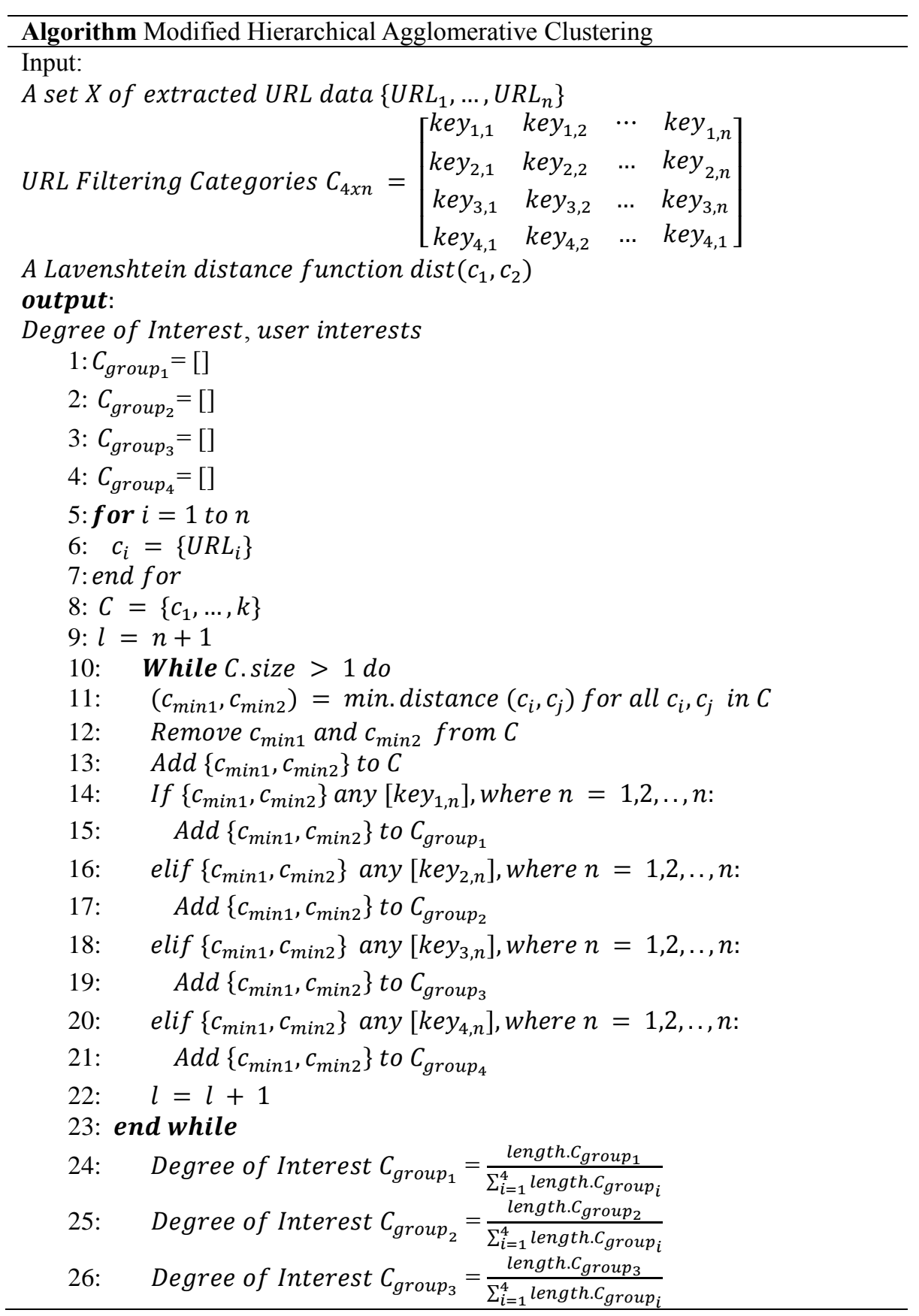


27: $\quad$ Degree of Interest $C_{\text {group }_{4}}=\frac{{\text { length. } \text { group }_{4}}_{\text {I }_{i=1}^{4} \text { length. }_{\text {group }}}}{\sum_{\text {28: }} \text { set } C_{\text {group }_{i}} \text { which has max (Degree of Interest) as user interests }}$

First is start by assigning each extracted URL data to a cluster, if we have $n$ URLs, it means we have $n$ clusters. Second, we compute the minimum distance between each cluster using Levenshtein distance function. Third, we find the closest (most similar) pair of clusters and merge them into a single cluster. Forth, we filter element of clusters using URL filtering categorizes. URL filtering categorizes will filter clusters based on keywords of users' interests. Fifth, we compute distances between the new cluster and each of the old clusters. We repeat steps 3, 4 and 5 until all extracted URL data has been clustered into a category of users' interests. After that we calculate interest degree for each category groups.

Table 2. URL filtering categories

\begin{tabular}{ll}
\hline Category Group & Category Type \\
\hline Business & $\begin{array}{l}\text { Business/Economy, Job Search/Careers, real } \\
\text { estate, and shopping }\end{array}$ \\
\hline $\begin{array}{l}\text { Communications } \\
\text { and search }\end{array}$ & $\begin{array}{l}\text { Blog/Web Communication, social networks, } \\
\text { email, and search engines /portals }\end{array}$ \\
\hline General & $\begin{array}{l}\text { Computer/Internet, education, news/media, } \\
\text { and reference }\end{array}$ \\
\hline Lifestyle & $\begin{array}{l}\text { Entertainment, games, arts, humor, religion, } \\
\text { restaurants/food, and travel }\end{array}$ \\
\hline
\end{tabular}

In our real work, we classify URL filtering categories into four main categories. The categories and their category type are shown on Table 2. Filtering categories consists of business category group, communications and search category group, general category group, and life style category group. The matrix $C$ of size $4 \times n$ to represent filtering categories can denoted as

$$
C_{4 x n}=\left[\begin{array}{llll}
\text { key }_{1,1} & \text { key }_{1,2} & \ldots & \text { key }_{1, n} \\
\text { key }_{2,1} & \text { key }_{2,2} & \ldots & \text { key }_{2, n} \\
\text { key }_{3,1} & \text { key }_{3,2} & \ldots & \text { key }_{3, n} \\
\text { key }_{4,1} & \text { key }_{4,2} & \ldots & \text { key }_{4, n}
\end{array}\right]
$$

Where rows represents category group of users' interests and columns represent keywords on each category group. We categorize the clustered results into category group based on keywords on matrix of filtering categories.

\section{Experimental Results}

In our study, we collected browsing history data of 30 smartphone users during one month continously. Browsing history data was tested on log files stored by the server. 
We extracted collected data and then use proposed algorithm which is called by Modified Hierarchical Agglomerative Clustering to provide smartphone user profile for interests-focused. In our experiment, we compare the performance between our method and C4.5 algorithm.

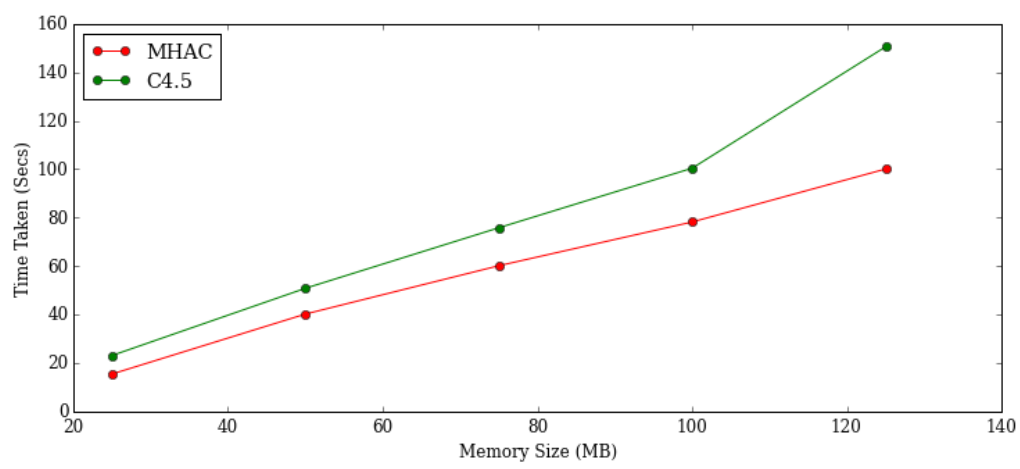

Fig. 1. Execution time comparison with $\mathrm{C} 4.5$ algorithm

Fig. 1 presents the execution time results of Modified Hierarchical Agglomerative Clustering algorithm (MHAC) and C4.5 algorithm. Our method consistently outperforms the C4.5 algorithm on all memory utilization in execution time. The results of degree of smartphone users' interests for each category are shown on Fig. 2. Finally, the results of smartphone user profile for interests-focused based on the highest degree for each user is shown on Fig. 3.

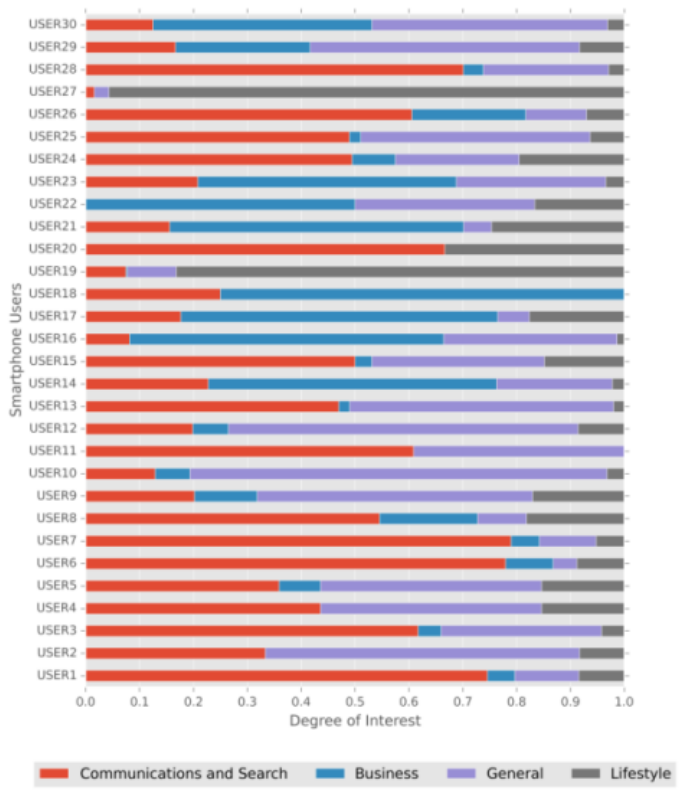

Fig. 2. Degree of smartphone user interests 


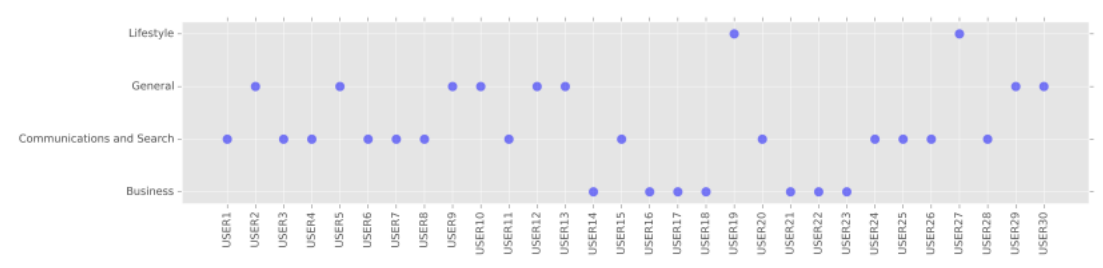

Fig. 3. Results of smartphone user profile for interests-focused

\section{Conclusion and Future Work}

In this paper, we have implemented a server-based application that can be used to provide user profile for interests-focused based on browsing history of web browser applications. In our approach, we propose a Modified Hierarchical Agglomerative Clustering to cluster extracted data which can automatically provide an interests profile of smartphone users. Based on experimental results, the proposed method can measure degree of users' interests based on browsing history of web browser applications, inferring particular pieces of information particular pieces of information that they interested on it, and outperforms the $\mathrm{C} 4.5$ algorithm in execution time on all memory utilization. Because amount of data that will be processed is increased, so in the future we need to implement Map-Reduce algorithm on Modified Hierarchical Agglomerative Clustering to enhance performance of clustering algorithm.

\section{Acknowledgements}

This research was supported by Basic Science Research Program through the National Research Foundation of Korea (NRF) funded by the Ministry of Education (2012R1A1A2007014).

\section{References}

[1] J. Hu, H.-J. Zeng, H. Li, C. Niu and Z. Chen, "Demographic Prediction Based on User's Browsing Behavior," in International World Wide Web Conference, Beijing, 2007.

[2] A. K. Santra and S. Jayasudha, "Classification of Web Log Data to Identify Interested Users Using Naïve Bayesian Classification," IJCSI International Journal of Computer Science Issues, vol. IX, no. 1, pp. 381-387, 2012.

[3] JinHuaXu and HongLiu, "Web User Clustering Analysis based on KMans Algorithm," in International Confrence on Infrmation, Networking and Automation (ICINA), HangZhou, 2010. 
[4] X. Min-jie and Z. Jin-ge, "Research on Personalized Recommendation System for eCommerce based on Web Log Mining and User Browsing Behaviors," in International Conference on Computer Application and System Modeling, ZhengZhou, 2010.

[5] N. Anand, "Effective Prediction of Kid's Behaviour Based on Internet Use," International Journal of Information and Computation Technology, vol. IV, no. 2, pp. 183-188, 2014.

[6] J. Li, "Research of Analysis of User Behavior Based on Web Log," in International Conference on Computational and Information Sciences, Anshan, 2013.

[7] S. Wang, L. She, Z. Liu and Y. Fu, "Algorithm Research on User Interests Extracting via Web Log Data," in International Conference on Web Information Systems and Mining, Chengdu, 2009.

[8] T. Murata and K. Saito, "Extracting Users' Interests from Web Log Data," in International Conference on Web Intelligence, Tokyo, 2006.

[9] W. McKinney, Python for Data Analysis, Gravenstein Highway North, Sebastopol, CA 95472.: O'Reilly Media, Inc, 2012.

[10] M. A. Russell, Mining the Social Web, Gravenstein Highway North, Sebastopol, CA 95472: O’Reilly Media, Inc, 2011.

[11] C. Rossant, Learning IPython for Interactive Computing and Data Visualization, Livery Place, 35 Livery Street, Birmingham B3 2PB, UK.: Packt Publishing Ltd, 2013.

[12] S. Vaingast, Beginning Python Visualization, New York: Springer-Verlag New York, Inc, 2009. 